\title{
Scolia
}

Revue de linguistique

\section{Claude MULLER, Indéfinis et partitifs en français}

\section{Emilia Hilgert}

\section{OpenEdition \\ Journals}

Édition électronique

URL : http://journals.openedition.org/scolia/1436

DOI : $10.4000 /$ scolia. 1436

ISSN : 2677-4224

\section{Éditeur}

Presses universitaires de Strasbourg

\section{Édition imprimée}

Date de publication : 10 juillet 2020

Pagination : 135-138

ISBN : 979-10-344-0067-6

ISSN : $1253-9708$

\section{Référence électronique}

Emilia Hilgert, «Claude Multen, Indéfinis et partitifs en français », Scolia [En ligne], 34 | 2020, mis en ligne le 10 juillet 2020, consulté le 25 septembre 2020. URL : http://journals.openedition.org/scolia/1436 ; DOI : https://doi.org/10.4000/scolia.1436 
SCOLIA 34/2020, p. 135-148.

Claude MULLER, Indéfinis et partitifs en français, Pessac, Presses universitaires de Bordeaux, 2019, 506 pages.

L'autorité de Claude Muller en matière d'indéfinis n'est plus à démontrer. Nous ne pouvons que saluer la synthèse qu'il nous livre dans son volume de 2019, regroupant ses analyses plus anciennes ou plus récentes de l'expression linguistique de l'indéfinitude, qui révèlent toute la complexité d'un domaine qu'il observe depuis quarante ans déjà. Les solutions proposées se situent dans un cadre générativiste et se structurent autour de deux points essentiels: la nature de de, outil remplissant de multiples fonctions dans la syntaxe des indéfinis, et le classement des indéfinis selon leurs domaines d'occurrence. La méthode est bien connue: dans un agréable style d'essai, très riche en illustrations et explications, qui nous permet de glaner souvent des pépites, l'auteur présente un examen minutieux des contextes de fonctionnement syntaxique des unités observées, livre une fine discussion des analyses déjà proposées et conclut par une présentation synthétique des résultats, par des définitions parfois évolutives, au fur et à mesure de l'analyse, et des réorganisations des domaines des indéfinis. Si certains titres se répètent ( Le de négatif», par exemple), c’est parce que les faits sont présentés d'abord dans une partie analytique, pour être repris dans une partie de solutions et propositions. Même si le parti pris est avoué comme sémantique (p. 28), l'auteur s'appuie principalement sur des critères syntaxiques, visibles dans l'étude initiale des contextes d'emploi, dans les manipulations qui doivent révéler la nature des constituants ou dans la représentation formelle des mécanismes profonds de fonctionnement.

Il n'est pas dépourvu d'intérêt de présenter ici quelques-unes des idées principales de ce volume. L'objet d'étude est donc la classe des déterminants avec ou sans nom et des pronoms saturés dont la propriété commune est l'indéfinition référentielle, c'est-à-dire sans référence par eux-mêmes: leur référence est essentiellement 
déterminée par le contexte, par la prédication dans laquelle ils entrent et qui produit la spécification d'un groupe nominal.

L'entrée en matière se fait par le de partitif, exploré par Claude Muller depuis les débuts de ses recherches linguistiques. L'auteur pose le problème des usages de de dans différentes structures, telles que de la bière, des bières, beaucoup de bière, une goutte de bière, (boire) de cette bière, un verre de cette bière, (ne pas boire) de bière, (ne pas boire) de cette bière, pas de bière, de nouveaux types de bière, ainsi que (j'écoute) du Mozart ou (c'est) du sérieux. Du point de vue morphologique, l'auteur analyse séparément de et le dans ce qu'on appelle traditionnellement "article partitif» et, en soumettant les structures observées aux opérations de dislocation et de cliticisation, donne son explication sur la nature de chacun des éléments. Ainsi choisit-il de considérer que de est dans tous ces cas la même préposition (en tous les cas historiquement), qui a des valeurs différentes en fonction des combinaisons syntaxiques où elle figure: des valeurs d'article, de relateur, de thématiseur, ou encore de partitif, valeurs qui interagissent ou qui s'additionnent en fonction des contextes (voir page 118, par exemple). Quant à le, deuxième élément de $d u$ et des, articles (alors que de seul peut aussi être un article), Claude Muller considère qu'il ne représente pas une classe générique, mais qu'il remplit une sorte de fonction métalinguistique en renvoyant à la classe de dénomination, servant de support morphologique pour distinguer le singulier du massif du pluriel du nombrable ou du collectif, étant donc un "classifieur» nominal.

C'est dans le cadre de l'analyse des valeurs de de que l'auteur différencie le partitif indéfini des syntagmes du cognac / de la bière du partitif défini des constructions un verre de ce cognac / de cette bière ou (boire) de ce cognac / de cette bière, à l'aide desquelles le locuteur rapporte l'objet choisi à un ensemble de référence. Dans ce deuxième contexte, de marque, pour Claude Muller, un cas partitif, étant donc une marque casuelle qui n'est pas sans rappeler, comme le fait l'auteur même, des constructions similaires en de du latin qui ont concurrencé le genitivus partitivus. L'opposition morphologique et interprétative entre le de partitif indéfini ou basique et le de partitif défini ne se fait que par la nature de leurs compléments. Claude Muller choisit donc de ne pas suivre la proposition de Milner (1978) quant à la distinction entre de quantifieur et de partitif, en faveur d'une analyse unitaire de de 
préposition, rappelant la proposition unifiante de Kupferman (2004), avec la précision que, si ce dernier propose une analyse unitaire en termes d'articles quantifieurs, Claude Muller insiste sur le fait que la sémantique et la syntaxe sont "profondément modifiées par le contexte du complément défini»: dans la gamme d'emploi de de, certains contextes rendent saillantes les propriétés de préposition de $d e$, d'autres les inhibent. La différence réside globalement dans l'opposition entre un article le «classifieur nominal» sans interprétation ensembliste dans le partitif indéfini et un article le défini «ensembliste» dans le cas du partitif défini.

Le deuxième grand objectif de Claude Muller est la présentation des indéfinis du type quelque, plusieurs, etc. De l'aveu de l'auteur, ce n'est pas le sens lexical qui l'intéresse prioritairement, mais les contextes ou environnements syntaxiques dans lesquels on peut ou on doit les utiliser. Cela ne surprend guère, l'un des premiers critères de son analyse est la polarité (négative ou non), combinée à la spécification. L'auteur ne se limite pas aux contextes assertifs basiques, mais cherche des illustrations de fonctionnement des indéfinis dans les questions, les constructions conditionnelles, les phrases impératives, la négation, les phrases générales, les compléments de comparatifs, etc. C’est de ces contextes d'usage que découlent les valeurs sémantiques de ces indéfinis: la spécification (qui mène à des spécifiés spécifiques et à des spécifiés non spécifiques), la quantification, la partitivité, le jugement de valeur (pour les dépréciatifs). Fort des résultats de ses analyses d'exemples attestés et relativement récents, Claude Muller rectifie l'étendue précise de chaque série ou sous-classe d'indéfinis proposée par Haspelmath (1997) et propose deux nouvelles cartes des domaines d'occurrence des indéfinis (cf. pages 331 et 434). Entre autres, il signale que la série des négatifs ne s'applique pas, en français actuel, au domaine des conditionnelles et des interrogatives. Il attire aussi l'attention sur le fait que le critère morphologique peut être trompeur, parce que de nombreux indéfinis ont des propriétés différentes au singulier et au pluriel (cf. quelque vs quelques) ou en tant que déterminants ou pronoms (cf. tous les vs tout), ce qui modifie les contours des sousclasses des indéfinis selon leur sens.

Quant à dresser une liste définitive des indéfinis, la tâche semble à Claude Muller délicate, parce que, si la grande majorité des formes observées réponde aux critères de l'indéfinition référentielle, quelques 
autres formes sont plus ambigües ou plutôt hybrides: tous les défini se voit modifier par la négation menant à une quantification indéfinie («pas tous»); de même, à côté de tous les défini, tout $N$ est bien indéfini; dans la série corrélative les uns et les autres, le premier est indéfini, malgré les marques morphologiques. La forme le même cache, encore une fois malgré l'article défini, un indéfini: un $N$ qui est le même. Claude Muller termine sur une note de sagesse (page 471): "[on] peut dès lors être plus indulgent pour la tradition grammaticale, qui ne peut se permettre de distinguer dans le détail de toutes les formes apparentées» de ces mots qui renvoient à des fragments à géométrie variable rapportés à une dénomination englobante, en insistant plutôt sur l'importance de l'étude des contextes d'emploi.

Emilia HILGERT

Université de Reims Champagne-Ardenne emilia.hilgert@univ-reims.fr

\section{Références}

HASPELMATH M. (1997), Indefinite Pronouns, Oxford, Oxford University Press.

KUPFERNAN L. (2004), Le mot «de». Domaines prépositionnels et domaines quantificationnels, Bruxelles, De Boeck-Duculot.

MILNER J.-C. (1978), De la syntaxe à l'interprétation, Paris, Éditions du Seuil. 
Jacques BRES, Aleksandra NOWAKOWSKA et Jean-Marc SARALE, Petite grammaire alphabétique du dialogisme, Paris, Classiques Garnier, coll. Domaines linguistiques, Série Formes discursives, 2019, 419 pages.

L'ouvrage intitulé Petite grammaire alphabétique du dialogisme (dorénavant $P G A D$ ) résulte d'une collaboration entre trois chercheurs de l'université de Montpellier, Jacques Bres, Aleksandra Nowakowska et Jean-Marc Sarale, qui ont chacun déjà produit des travaux dans le champ des études sur le dialogisme et l'analyse de discours, avec des inflexions respectives dans les domaines des temps verbaux, de l'argumentation, de la syntaxe et de la lexicologie, dont beaucoup de problématiques se trouvent déclinées dans plusieurs entrées du dictionnaire. Celui-ci se présente ainsi comme une sorte de manuel à destination d'un public très large: « chercheurs et enseignants en sciences du langage, en sciences de l'information et de la communication, en lettres, en traductologie ou en didactique, et à tous les chercheurs en sciences humaines qui se confrontent aux discours» (p. 28), d'où sa présentation alphabétique réputée plus maniable (p. 10). Son ambition est de constituer un "outil indispensable à l'analyse du discours" (p. 28). Même s'il est le fruit d'un travail collectif, on trouve à la fin de l'Introduction (p. 29) une liste des entrées principalement traitées par chacun des contributeurs.

Le projet de constituer un tel «outil de référence» (p. 9) provient, de l'aveu même des auteurs, du sentiment d'une lacune dans le champ de l'analyse de discours. Bien qu'on s'y réfère en effet très souvent au «dialogisme», «la notion [...] n'a pas à ce jour été traitée dans le détail linguistique de son fonctionnement» (ib.), gageure que les contributeurs entendent donc précisément relever par leur ouvrage. Le terme de grammaire est justifié à plusieurs reprises par le fait que le phénomène est "abordé à partir de ses marques»(p.12), qu’il s'agisse d'un marqueur proprement dit - «élément qui est la trace en discours d'opérations énonciatives stables» (p. 23) - ou d'un signal "élément qui contribue sporadiquement, en contexte, à l'expression du dialogisme sans que son signifié en langue prédise nécessairement cet emploi»(ib.). Quoique la distinction entre ces deux types de marques ne soit pas systématique dans le cours de l'ouvrage, le terme de marqueur apparaissant bien souvent comme l'hyperonyme de la catégorie $(i b$.$) , la démarche consiste en somme à partir de faits$ 
linguistiques «qui pour l'essentiel sont des objets traditionnels des grammaires de langue, comme la négation, le clivage, les déterminants ou les temps verbaux» (p. 10) pour remonter vers la "potentialité dialogique» du fait en question, située à «l'articulation de la langue et du discours» (p. 154); ainsi de celle de l'article défini (p. 41, 43, 45), $\mathrm{du}$ déterminant démonstratif (p. 109, 122) ou du passif (p. 325) par exemple. Certaines entrées s'écartent pourtant de ce parti pris grammatical en s'inscrivant dans une perspective plus rhétorique ou argumentative - la confirmation, la glose de mot, la modalisation par discours autres, la reformulation -, voire franchement stylistique - le détournement, le discours rapporté, l'écho, l'hypochorisme, l'insistance pronominale, l'ironie, le parallélisme, la prolepse, le renchérissement; au point que le terme de grammaire dans le titre pourrait en paraître quelque peu forcé. Tel n'est pourtant pas nécessairement le cas si l'on observe que la distinction entre le "dialogisme en langue» et le "dialogisme en discours", invoquée à plusieurs reprises dans l'ouvrage (par exemple p. 95, 154-158, 232), pour peu qu'elle ne soit pas explicitement fondée - si elle peut l'être -, aboutit à mettre sur le même plan des phénomènes relevant de niveaux n'appartenant pas a priori aux mêmes plans de description. Il en résulte une certaine impression d'arbitraire dans le choix des entrées retenu: puisqu'on peut a priori «voir du dialogisme partout» (p. 57), quel a été le principe de sélection des faits étudiés, dont les auteurs déclarent avoir traité les "principaux» sans pour autant prétendre à l'exhaustivité (p. 27)? Et si tous les faits de langue, pour s'en tenir à eux, ne relèvent pas au même degré du dialogisme, cette gradation gagnerait à être fondée aussi systématiquement dans tous les cas que dans celui qui oppose «conditionnel et futur» dans l'entrée du même nom (p. 95-102), faute de quoi on en revient à ce concept tellement général qu'il en devient inopérant de "potentialité dialogique».

Les auteurs définissent d'emblée le dialogisme comme un principe consistant en "l'orientation de tout discours [...] vers d'autres discours", qui "se réalise sous forme de dialogue interne avec ceux-ci» (p. 10). Le dialogisme est compatible avec tous les genres de discours, contrairement à la dimension dialogale réservée au dialogue, «marqué par l'alternance des tours de parole de différents locuteurs» (p. 11). Ce dialogisme peut donc réagir à un discours antérieur (dialogisme interdiscursif), au discours de l'allocutaire (dialogisme interlocutif) 
ou réflexivement à lui-même (dialogisme intralocutif); ces catégories étant systématiquement déclinées au fil des différentes entrées du dictionnaire grâce à de nombreux exemples authentiques (4000 selon les auteurs: p. 27) pris dans des genres et des formations discursives variés, quoique de manière à la longue un peu mécanique. Cette notion est en un sens plus large que celle que recouvre la dénomination en partie concurrente de "polyphonie», puisqu'elle englobe avec le dialogisme interdiscursif des phénomènes relevant de l'intertextualité, qu'il s'agisse de l'intertexte proprement dit ou de l'interdiscours (p. 12-13). De même le discours rapporté direct se voit intégré tel quel au dialogisme, là où Ducrot différencie la polyphonie induite par le «rapport en style direct» de la "double énonciation» proprement dite, que ce dernier auteur considère comme un cas beaucoup plus fréquent de polyphonie (1984: 203). C'est que les auteurs de la $P G A D$ ont supprimé un étage du dispositif énonciatif proposé par ce même Ducrot (1984): le sujet parlant - le producteur empirique du message verbal - de celui-ci correspond en effet au locuteur de ceux-là (24), son locuteur - l'être de discours auquel réfèrent les marques de première personne - est leur énonciateur (ib.), et partant son énonciateur - être dont l'énonciation exprime le point de vue, la position, l'attitude, «mais non pas, au sens matériel du terme, [les] paroles» (Ducrot op. cit.: 204) - ne trouve pas place dans l'architecture du dialogisme, ce qui a pour conséquence de rendre sans objet - et partant sans entrée - toute la problématique du "point de vue» en narratologie et ailleurs (Rabatel, 2008; voir la discussion avec Nølke et la ScaPoLine dans Ducrot, 2001), omission qui a de quoi surprendre chez des chercheurs se réclamant aussi continûment de Bakhtine (p. 9-11). Cette position est cependant cohérente avec la réserve manifestée vis-à-vis de l'emploi métaphorique du terme de voix, auquel les auteurs substitueraient volontiers systématiquement le terme de discours (p.25), ce qui ramène le "dialogisme», entendu au sens de la $P G A D$, à la seule "première polyphonie» de Ducrot, celle du discours rapporté (1984: 196-197), entendu «non seulement comme ce qui est effectivement dit, mais aussi comme ce qui a pu être dit et ce qui pourra être dit; c'est-àdire dont la matérialité n'est pas liée strictement à des mots précis, à des énonciations effectives, mais consiste en un ensemble de formulations en relation paraphrastique qui domine les locuteurs-énonciateurs» (p. 25). En résulte un traitement de l'ironie comme «interaction 
particulière avec le discours antérieur d'un locuteur-énonciateur cible» (p. 255), tandis qu'elle est conçue par d'autres linguistes comme «exprimant la position d'un énonciateur E, position dont on sait par ailleurs que le locuteur L n'en prend pas la responsabilité, et bien plus, qu'il la tient pour absurde» (Ducrot, 1984:211, cf. aussi Berrendonner, 1981: 173-239). De même, des deux négations «conflictuelles» posées par Ducrot (1981), la négation métalinguistique et la négation polémique, n'est abordée dans l'ouvrage que la première, dans la mesure où seule celle-ci infirme une énonciation antérieure effective (entrée «Négation prédicative», p. 273-281).

En s'opposant aux approches «polyphoniques» d'inspiration pragmatique (p. 26) la $P G A D$ présente une conception alternative du phénomène dialogique qui fournirait matière à la méthodologie de la linguistique, car elle met en question «l'idée, effectivement reposante, selon laquelle on peut dissocier un phénomène et la façon dont on le traite, le phénomène restant identique à lui-même sous les différents traitements qu'on lui fait subir» (Ducrot, 2001). Pour autant, une nouvelle dénomination n'est pas nécessairement le gage d'un nouvel objet scientifique. Ainsi ce que les auteurs désignent comme le dialogisme interlocutif anticipatif (p. 14) semble-t-il bel et bien correspondre à la diaphonie potentielle de Roulet et al. (1985: 83); de même, le "fonctionnement dialogique» du déterminant démonstratif (p. 108) n'est pas sans évoquer l'exophore mémorielle naguère décrite par Bordas (2001) ou Viprey (2006) à la suite de Fraser et Joly (1980) et de Weinrich (1973); quant au «fonctionnement interlocutif de la dislocation gauche», il a été décrit comme "entrée-préface» chez Perrin (1999), qui fait dans le même article une large place à la question de l'Écho (réponse écho positive ou négative dans le dialogue). Même si des considérations matérielles conduisent à limiter la place accordée aux références (p. 27), on regrettera dans le même ordre d'idée que la Grammaire de la période (2013) ne soit pas non plus citée aux entrées de la «Dislocation» ou du "Pseudo-clivage», dont elle étayerait pourtant notablement les analyses. De telles résonances rendraient certainement encore plus dialogique la $P G A D$, laquelle se présente déjà, telle quelle, comme une contribution aux débats sur cette question riche et complexe. 


\section{Références}

BERRENDONNER A. (1981), Éléments de pragmatique linguistique, Paris, Minuit.

BORDAS É. (2001), Un stylème dix-neuviémiste: Le déterminant discontinu un de ces... qui..., L'Information Grammaticale 90, 32-43.

DUCROT O. (1984), Le dire et le dit, Paris, Minuit.

DUCROT O. (2001), Quelques raisons de distinguer «locuteurs» et «énonciateurs", Polyphonie linguistique et littéraire 3, 20-41.

FRASER T. \& JOLY A. (1980), Le système de la deixis (2): endophore et cohésion discursive en anglais, Modèles linguistiques II: 2, 22-51.

GROUPE DE FRIBOURG (2012), Grammaire de la période, Berne, P. Lang.

PERRIN L. (1999), La fonction des reprises diaphoniques locales dans le dialogue, in Verschueren J. (éd.), Pragmatics in 1998, vol. 2, 448-461, Anvers, IPrA.

RABATEL A. (2008), Homo narrans, t. II, Dialogisme et polyphonie dans le récit, Limoges, Lambert-Lucas.

ROULET E. \& AL. (1985), L'articulation du discours en français contemporain, Berne, P. Lang.

VIPREY J.-M. (2006), Un de ces [syntagmes] qui... (à propos de la locution un(e) de ces [...] qui), Corpus, 5, <http://journals. openedition.org/corpus/713>, consulté le 30 avril 2019.

WEINRICH H. (1973), Le Temps, Paris, Seuil. 
Bert PEETERS (ed.), Heart-and Soul-Like Constructs across Languages, Cultures, and Epochs, 2019, New York and London, Routledge Taylor \& Francis Group, 148 pages.

L'ouvrage collectif édité par Bert Peeters sous le titre, Heart-and Soul-Like Constructs across Languages, Cultures, and Epochs (Cour-et Ame-comme constructions en plusieurs langues, cultures et époques) s'inscrit parmi les études des valeurs culturelles saillantes et des mots qui les désignent dans différentes langues, dans la perspective méthodologique de la Métalangue Sémantique Naturelle - MSN (Natural Semantic Metalanguage - NSM). Il fait ainsi suite au numéro thématique de la revue International Journal of Language and Culture de 2015 (volume II, $n^{\circ}$ 2), édité par le même auteur, consacré aux liens entre langage et valeurs culturelles.

L'approche du langage MSN, initiée par Wierzbicka (1996, 1997, 2014) et développée avec ses collaborateurs, en particulier avec Goddard (2011, 2018), approche que Peeters définit comme «a powerful tool consisting of a maximally culture-neutral vocabulary and $\operatorname{syntax}[\ldots] »($ p. 7) trouve dans cet ouvrage une illustration à la fois fidèle et innovante sur le plan de l'argumentation de ses concepts et de ses évolutions.

Dans le premier chapitre de l'ouvrage, intitulé Delving into Heartand Soul - Like Constructs (Étudier/ fouiller/ entrer dans le Cour et l'Âme comme des constructions), Peeters s'attache justement à présenter, expliciter et argumenter aussi bien la dernière version de l'approche MSN, que ses évolutions: l'évolution de la liste des primitifs sémantiques en anglais et dans d'autres langues, l'évolution des «explications» de la signification des mots, de l'organisation syntaxique des primitifs sémantiques et des molécules mobilisés pour bâtir cette explication, propre à chaque langue et culture. On parle d'explication, car le terme de description sémantique ne correspond pas à l'objectif de l'approche MSN qui vise à faire comprendre des concepts saillants et les mots qui les désignent dans une langue et culture aux sujets parlants d'autres langues et cultures. Il s'agit de concepts et de mots d'une grande complexité et d'une haute spécificité culturelle, comme les valeurs culturelles et les «Ethnopsycological Personhood Constructs» (désormais EPCs), dont la traduction s'avère en réalité impossible. La technique proposée est celle connue sous le nom de 
"paraphrase réductive» en termes sémantiquement simples. Ces termes, les primitifs sémantiques, dont la liste est limitée actuellement à 65 items, et les molécules, appelées à compléter la paraphrase réductive là où les primitifs ne suffisent pas, permettent de produire une explication susceptible de faire apparaître les ressemblances et les différences culturelles.

On peut adhérer ou non à cette approche, le chapitre a le grand mérite de la présenter et de la conforter par des arguments liés à son application et par des illustrations, d'une manière qu'on pourrait presque qualifier de didactique. Chaque élément est repris et discuté dans son évolution.

On pourrait s'interroger sur l'absence de discussion des fondements théoriques de cette approche du sens linguistique, mais le chapitre proposé par Bert Peeters est orienté explicitement vers la puissance explicative du modèle MSN, d'une culture à l'autre, d'une langue à l'autre et l'objectif de cet ouvrage est justement de justifier ce modèle dans un champ sémantique particulièrement sensible culturellement: celui des EPCs. L'auteur présente et explicite d'abord ce champ sémantique en faisant aussi l'historique et l'état de l'art pour chacun des éléments de cette expression (voir aussi Levisen, 2017).

En fait, l'idée centrale de l'ouvrage est que la MSN, surtout dans sa version actuelle, est susceptible de rendre compte des spécificités culturelles des concepts ethnopsychologiques et donc des mots qui les désignent. Il s'agit notamment de concepts et de mots se rapportant à la partie non visible de la personne, siège des pensées, doutes, intentions, désirs, affects, vus comme des constructions ancrées culturellement. Et ce faisant, de sortir de l'anglocentrisme qui fait que, par exemple, le concept de "mind" soit dominant jusque dans les approches scientifiques, par rapport à des concepts comme $d u s ̌ a$, en russe ou esprit en français, etc. Pour éviter justement ce processus de «réification" d'un concept comme «mind", l'explication doit porter sur ce que ce concept a en commun avec les conceptualisations de la partie non visible de la personne siège de pensées, croyances, affects, etc. dans d'autres langues et cultures et sur ce qui l'en distingue.

C'est là que réside la grande cohérence de l'ouvrage qui réunit des études sur: inochi («life») et tamashii («soul») en japonais (Yuko Asano-Cavangh, chapitre II), anoa («spirit») en Longgu, une langue océanique parlée dans les Iles Salomon (Deborah Hill, chapitre III), 
la construction du concept de chai («heart») en langue thaï (Chavalin Svetanant, chapitre IV) et le mot hugr (comme "mind», "heart» et «courage») en vieux scandinave (Colin Mackenzie, chapitre V).

Seul le chapitre II n'utilise pas la dernière version NSM, plus précisément l'idée de "patron sémantique» (semantic template) qui assure une organisation, une structure sémantico-syntaxique intrinsèque des primitifs et molécules sémantiques convoqués dans l'explication du concept porté par le mot étudié. Par ailleurs, ce chapitre construit l'étude de inochi et de tamashii comme une réponse aux questionnements sur l'intelligence artificielle référée à l'intelligence humaine et au langage. Dommage toutefois que la réponse ne soit pas référée et argumentée aussi avec la célèbre réponse de John Searle sur la «chambre chinoise» (Searle, 1980). En revanche, on ne peut que souligner l'itération remarquable entre la convocation des études anthropologiques et ethnologiques de la culture japonaise et l'analyse linguistique, explicative et comparative des concepts et des mots. Autrement dit, la réflexion du linguiste sur les concepts portés par les significations des mots se nourrit de et nourrit à son tour la réflexion des anthropologues et des ethnologues sur la culture japonaise.

La même itération remarquable entre les disciplines qui se croisent dans l'approche des EPCs est présente dans le chapitre III. L'ancrage culturel de anoa se décline dans les fondements religieux, et plus largement dans une conception ancestrale qui relie le corps et l'esprit, selon un mouvement du second vers le premier, la personne humaine étant conceptualisée "from the Inside Out», de l'esprit vers le corps.

Les auteurs des chapitres IV et V s'attachent à justifier leurs «explications» linguistiques (Chavalin Svetanant) leurs explications et/ ou scripts culturels (Colin Mackenzie) par une très sérieuse étude de corpus: Thaï National Corpus (TNC), id est 32667991 mots tirés de textes écrits et The National Dictionnary of the Thai Royal Institute B.E. de 2011, pour l'étude de chai en langue thaï, et le Dictionary of Old Norse Prose (DONP) de l'Université de Copenhague pour l'étude du EPC hugr.

Il semble important de souligner que cette démarche ajoute à l'introspection et aux enquêtes introspectives, très présentes dans l'approche MSN, une étude de corpus écrits fournissant des exemples et des illustrations pour chaque élément présent dans les explications de Svetanant et dans les scripts culturels et les explications de Mackenzie. 
Fournissant aussi et surtout des arguments, avec ces exemples attestés, en faveur de leurs choix méthodologiques: le sens commun et des évaluations chez Mackenzie et une bonne illustration de la puissance de translation d'une langue à l'autre, par la formulation des explications MSN en même temps en anglais et en thaï chez Svetanant.

Pour conclure, on peut revenir sur la défense et l'illustration de l'approche MSN dans sa version actuelle, proposées par Bert Peeters avec cet ouvrage collectif. Attentif à la dynamique de cette approche, à sa puissance explicative quand il s'agit de comparer et de contraster les EPCs des différentes langues et cultures, à l'appréhension de l'explication comme une forme d'expérimentation perfectible, l'éditeur assure la cohérence de sa démarche en insérant, à la fin de chacun des chapitres des contributeurs, un commentaire. Il fait ainsi apparaître les éventuels "écarts» de leurs approches par rapport à la version MSN présentée et défendue dans le chapitre introductif, "écarts» ou «retours» vers une version plus ancienne, par rapport auxquels il donne son point de vue, ouvrant ainsi un débat et une piste de recherche et d'approfondissement du modèle. On peut souhaiter que cette ouverture puisse se faire aussi à l'avenir, par un positionnement parmi d'autres approches du sens linguistique appréhendé dans son ancrage culturel, comme construction du monde référentiel, certes d'abord dans la zone des EPCs et des valeurs culturelles, mais aussi dans d'autres zones sémantiques du fait social et humain.

Olga GALATANU

Université de Nantes olga.galatanu@univ-nantes.fr

\section{Références}

GODDARD C. (2011 [1998]), Semantic Analysis: A practical Introduction, Oxford, Oxford University Press.

GODDARD C. (2018), Ten lectures on Natural Semantic Metalanguage: Exploring language, thought and culture using simple, translatable words, Leiden, <Brill.doi:10.1163/9789004357723>.

LEVISEN C. (2017), Personhood constructs in language and thought: New evidence from Danish, in Zhengdao Ye (ed), The semantics of nouns, Oxford, Oxford University Press, 120-146. 
WIERZBICKA A. (1996), Semantics: Primes and Universals, Oxford, Oxford University Press.

WIERZBICKA A. (1997), Understanding Cultures Through Their Key Words: English, Russian, Polish, German and Japanese, New York/Oxford, Oxford University Press.

WIERZBICKA A. (2014), Imprisoned in English: The hazards of English as a default language, New York, Oxford University Press, <doi:10.1093/acprof:oso/978199321490.001.0001>. 
SCOLIA 34/2020, p. 149-153.

\title{
Abstracts/Résumés
}

\section{Elena Callegari, Université de Oslo Espen Klævik-Pettersen, Université de Oslo \\ Les sujets disloqués en trévisan. Étude pragmatique Dislocated Subjects in Trevisan. A Pragmatic Study}

\begin{abstract}
This paper looks at the discursive function of left-dislocated subjects in an understudied Romance variety, namely Trevigiano. In the interest of comparison, we also consider Ashby's (1988) corpus on left dislocations in French. We argue that left dislocated subjects in Trevigiano, just like their French counterparts, are topics which serve to activate a new file card in the sense of Heim (1982), and we show that the majority of left-dislocated subjects in Trevigiano introduce a topic shift at the level of the sentence, rather than at the level of the discourse. We then provide examples of how speakers exploit this flexible tool in order to develop and manipulate the different levels of discourse.
\end{abstract}

Keywords-. left dislocation, French, Trevigiano, pragmatics, topics, file card theory

Résumé-. Le présent article considère la fonction discursive des sujets disloqués à gauche dans une variété romane peu étudiée, à savoir le trévisan. Par souci de comparaison, nous avons également recours au corpus d'Ashby (1988) sur les dislocations en français parlé. Nous soutenons que les sujets disloqués en trévisan, tout comme en français, sont des topiques dont la fonction pragmatique est d'activer un nouveau fichier dans le sens de Heim (1982), et nous démontrons que la majorité des sujets disloqués en trévisan introduisent un transfert de topique au niveau de la proposition et non pas au niveau du discours. Nous fournissons des exemples démontrant comment les locuteurs exploitent cet outil flexible pour développer et gérer les différents niveaux du discours.

Mots-clés-. dislocation à gauche, francais, trévisan, pragmatique, topiques, théorie des fichiers 
Thématisation et périphéries de la phrase

\title{
Mathieu Goux, Université de Caen
}

\section{Commencer en continuant: du rôle des constructions détachées en -ant en tête de chapitre. L'exemple d'Oudin, de Rosset et de Vaugelas}

\author{
Starting by Continuing: The Role of -ant Detached Constructions at the \\ Beginning of Chapters. The Example of Oudin, Rosset, and Vaugelas
}

\begin{abstract}
We analyse the -ant detached constructions at the beginning of chapters of Oudin and Rosset's translation of Don Quixote by Cervantes (1614 and 1618), and Vaugelas' translation of Histories of Alexander the Great by Quinte-Curce (1653). We study the role of these structures in terms of textual cohesion, coherence and connexity. We also study their specificities in relation to other forms of informational progression in the classical era, while these -ant constructions undergo a significant decrease in frequency. At last, we offer an overview of the Latin and Spanish structures that these constructions translate, in order to better understand their use in classical French.
\end{abstract}

Keywords-. detached construction, cohesion, coherence, connexity, secondary predication, reference

Résumé-. Notre article analyse les constructions détachées en -ant en tête des chapitres de la traduction du Don Quichotte de Cervantès par Oudin et Rosset (1614 et 1618) et de la Vie d'Alexandre de Quinte-Curce par Vaugelas (1653). Nous étudions le rôle de ces structures au regard des notions de cohésion, de cohérence et de connexité textuelles, et leurs spécificités au regard d'autres formes de progression informationnelle à l'époque classique, tandis que ces constructions en -ant subissent une diminution fréquentielle marquante. Nous proposons également un panorama des structures latines et espagnoles que ces constructions traduisent, afin de saisir au mieux leur emploi en français classique.

Mots clés-. construction détachée, cohésion, cohérence, connexité, prédication seconde, référence

Hans Petter Helland, Université d'Oslo

Christine Meklenborg-Nilsen, Université d'Oslo

Terje Lohndal, NTNU, Trondheim \& Université de Tromsø

\section{La topicalisation en français et en norvégien}

\section{Topicalization in French and Norwegian}

Abstract-. In this paper, structural and syntactic properties of left dislocation as topics in French and Norwegian are examined in a systematic manner. We establish a standard division of Hanging Topics and Left Dislocated Topics and argue for the distinction in both languages. There are however fundamental structural differences between French and Norwegian related fundamentally to the V2 feature of Norwegian as a Germanic language. This means that we find language-specific characteristics. 
Abstracts / Résumés

We propose a movement approach for Norwegian, whereas French has both base generated Hanging Topics and Left Dislocations. In this manner we can explain typical differences between the two languages and refine insights that have been established in the linguistic literature for French.

Keywords-. movement and base-generation, topics, Hanging Topics, Left Dislocation, left periphery, interface syntax pragmatics, verb second

Résumé-. Nous examinerons dans cette contribution des différences structurales et syntaxiques entre des procédés de topicalisation en français et en norvégien. L'illustration de tels mécanismes se fait sur la base de dislocations à gauche qu'il s'agisse de Hanging Topics (nominatifs pendants) ou de dislocations à gauche au sens technique du terme. II y a des différences structurales fondamentales entre les deux langues, ce qui s'explique entre autres par les caractéristiques du norvégien comme une langue Verbe second (V2). Nous basant sur des propriétés distinctes de la topicalisation entre les deux langues, il nous est possible d'expliquer pourquoi le français se comporte différemment du norvégien tout en maintenant la division classique entre les deux types de dislocations.

Mots clés-. dérivation par mouvement, position de base, hanging topics, dislocation à gauche, périphérie gauche, interface syntaxe-pragmatique, verbe second

\section{Morgane Jourdain, KU Leuven, Université de Lille Karen Lahousse, KU Leuven}

\section{Les sujets disloqués et non-disloqués chez l'enfant français Dislocated and Non-Dislocated Subjects in Child French}

Abstract-. The aim of the present paper is to identify the contexts in which French children produce dislocated and non-dislocated subjects. For that purpose, we carried out a corpus study for 21 children between age 2 and 5 from the TCOF corpus, and extracted a total of 370 dislocated and 1029 non-dislocated subjects. We will show that, in child language, dislocated subjects (i) always correspond to the topic of the utterance, (ii) can express a contrastive topic and (iii) are used for referents which are less accessible for the interlocutor than non-dislocated subject pronouns. Lexical nondislocated subjects are extremely rare, as we found only 11 instances of such subjects. However, almost all of them appear in a very different context from dislocated subjects: they are typically produced in all-focus utterances. These results suggest that children master the functions of dislocation from the onset of the acquisition of the construction. This result puts in question former studies claiming that the clitic pronoun in child language is a morphological marker and hence a compulsory element in French child grammar.

Keywords-. Information structure, language acquisition, dislocation, syntax, topic

Résumé-. Le but du présent article est d'identifier les contextes dans lesquels les enfants français produisent des sujets disloqués et non disloqués. Pour cela, nous avons effectué une analyse de corpus pour 21 enfants entre 2 et 5 ans du corpus 
TCOF, et avons extrait un total de 370 sujets disloqués et 1029 sujets non-disloqués. Nous montrons que, chez l'enfant, les sujets disloqués (i) correspondent toujours au topique de l'énoncé, (ii) peuvent exprimer un topique contrastif et (iii) sont utilisés pour des référents moins accessible pour l'interlocuteur que des sujets pronominaux non-disloqués. Les sujets lexicaux non-disloqués sont très peu nombreux, 11 au total. Cependant, la quasi-totalité de ces cas apparaissent dans un contexte radicalement opposé aux sujets disloqués et pronominaux : ils sont produits dans des énoncés toutfocus. Ces résultats suggèrent que les enfants maîtrisent les fonctions de la dislocation dès le début de la production de cette construction. Cela qui remet en question les études antérieures selon lesquelles le pronom clitique chez l'enfant est un marqueur flexionnel et donc un élément obligatoire de la phrase dans la grammaire de l'enfant.

Mots-clés-. structure de l'information, acquisition du langage, dislocation, syntaxe, topique

\title{
Pierre Larrivée, Université de Caen
}

\section{Le focus initial en français vernaculaire}

\section{The Initial Focus in Vernacular French}

\begin{abstract}
It is generally assumed that the broad categories of syntax are available in every language. However, each language may vary in the way that they realize these categories, in the subset of values associated to their realizations and in their frequency. The category of Focus is realized in several languages by constructions such as clefts and initial focused phrases such as the French and Italian equivalent of Paul he's called. Focus Movement has a diversity of values that are systematically analyzed. The relative paucity of the configuration is proposed to be a consequence of the prosodic constraints of the language that discourages pre-verbal stress, following Lambrecht (1994).
\end{abstract}

Keywords-. focus movement, French, clefts, push chains, prosodic constraints

Résumé -. On suppose mutatis mutandis que les grandes catégories syntaxiques sont disponibles dans chaque langue. Pourtant, chaque langue peut varier dans la façon dont elle réalise ces catégories, dans les valeurs particulières que ces réalisations profilent et dans leur fréquence d'usage. La catégorie de Focus est ainsi actualisée dans diverses langues par une construction clivée, au côté de laquelle on retrouve des configurations à Focus initial de type Paul il s'appelle. L'étude de cette configuration est l'occasion de définir les valeurs multiples associées à la notion de Focus dans l'usage. La rareté relative du Focus initial en français est attribuée aux contraintes prosodiques de la langue décourageant l'accentuation préverbale, suivant l'intuition de Lambrecht (1994).

Mots-clés-. focus initial, français, clivées, chaîne de propulsion, contraintes prosodiques 
Abstracts / Résumés

\title{
Rakel Österberg, Université de Stockholm
}

\section{La périphérie gauche et droite dans la production des locuteurs natifs et non-natifs de français et chez les locuteurs natifs de suédois \\ The Left and Right Periphery in Native and Non-Native Speakers of French and Native Speakers of Swedish}

\begin{abstract}
Romance languages, including French, tend to put much information in the left periphery whereas in Swedish the trend seems to be the opposite (Morel \& Danon-Boileau, 1998, Conway, 2005, Hancock, 2007, Fant, in press). The first objective is to provide a global overview of the taxonomy of the constituents of the left and right periphery in French and Swedish L1, in order to afford a comparative analysis. The second objective is to compare French L2 usage with that of French L1. The results indicate significant differences between the patterns employed in the syntactic peripheries in French and Swedish. Swedish speakers tend to use the right periphery significantly more than L1 French speakers. As far as the left periphery is concerned, the production of French speakers far exceeds that of Swedish speakers. Advanced speakers of French L2 approach French L1 except regarding the most complex left peripheries.
\end{abstract}

Keywords-. left periphery, right periphery, pragmatic functions, French L1, French L2, high-level L2 proficiency

Résumé-. Les langues romanes, y compris le français, tendent à placer beaucoup d'informations dans la périphérie gauche tandis qu'en suédois la tendance semble aller dans le sens contraire (Morel \& Danon-Boileau, 1998 ; Hancock, 2007; Conway, 2005 ; Fant, sous presse). Le premier objectif de la présente étude est de fournir un aperçu global de la taxinomie des constituants des périphéries gauche et droite en français et suédois $L 1$, afin de permettre une analyse comparative. Le deuxième objectif est de comparer l'emploi en français $L 2$ à celui du français $L 1$. Les résultats montrent des différences importantes entre les patrons employés dans les périphéries syntaxiques en français et suédois. Les locuteurs suédois tendent à employer la périphérie droite beaucoup plus que les locuteurs de français L1. La situation s'inverse en ce qui concerne la périphérie gauche. Les locuteurs avancés de français $L 2$ présentent des caractéristiques comparables à celles du français $L 1$, sauf pour les périphéries gauches les plus complexes.

Mots-clés-. périphérie gauche, périphérie droite, fonctions pragmatiques, français $L 1$, français $L 2$, compétence très avancée d'une L2 